\title{
Assessment of IgH PCR strategies in multiple myeloma
}

R G Owen, R J Johnson, A C Rawstron, P A Evans, A Jack, G M Smith, J A Child, G J Morgan

\begin{abstract}
Aims-To compare the ability of four commonly used PCR techniques to demonstrate clonal IgH rearrangements in multiple myeloma.

Methods-Bone marrow samples (containing a minimum of $10 \%$ plasma cells) were obtained from 127 patients with confirmed multiple myeloma. Framework 3 (Fr3) PCR was performed in all cases and the Framework 1 (Fr1f) PCR, which utilises six VH family specific primers, in 98 cases. In addition, 44 cases were assessed by Fr3, Fr1f, Framework 2 (Fr2) and Framework 1 consensus (Fr1 con) PCR techniques. JH primer selection was also assessed such that each PCR strategy was performed twice in each of the 44 cases, using the $\mathrm{JH}$ consensus primer $\mathrm{JH}$ con) alone and then repeated with an equimolar mixture of $\mathrm{JH}$ con, $\mathrm{JH} 3$ and $\mathrm{JH} 6$ (JH mix).
\end{abstract}

Results-Clonal rearrangements were demonstrated in $71(56 \%)$ of 127 cases with the Fr3 PCR and in $52(53 \%)$ of 98 with the Fr1f PCR. However, by using both techniques it was possible to demonstrate clonal IgH rearrangements in $92 \mathbf{( 7 5 \% )}$ of 122 cases. Forty four cases were assessed by all four PCR techniques; in these cases the Fr3 and Fr1f PCRs demonstrated clonal rearrangements in $26(59 \%)$ cases with a combined yield of $34(77 \%)$. The Fr2 and Fr1 con PCR techniques had inferior pick up rates, demonstrating clonal rearrangements in $21(48 \%)$ of 44 cases and a combined yield of $28(63 \%)$. The Fr2 PCR did, however, demonstrate a clonal rearrangement in one case negative by both Fr3 and Fr1f. Two additional rearrangements were demonstrated by using $\mathrm{JH}$ mix; one became positive by Fr3, Fr1f and Fr2 and the other positive by Fr1f, Fr1 con and Fr2.

Conclusion-By utilising both the Fr3 and Fr1f PCR techniques it is possible to demonstrate definitive clonal rearrangements in the majority of patients with multiple myeloma. The Fr1 con and Fr2 PCR techniques have inferior pick up rates but may detect some additional rearrangements. (F Clin Pathol 1996;49:672-675)

Haematological Oncology, Institute of Pathology, The General Infirmary at Leeds, Great George Street, Leeds LS1 3EX

Correspondence to Dr G J Morgan.

Accepted for publication 4 June 1996

Keywords: immunoglobulin heavy chain gene, myeloma, PCR.

The advent of autologous and allogeneic transplantation as treatment options in multiple myeloma has resulted in a necessity for $\frac{\overline{\bar{D}}}{\vec{D}}$ molecular monitoring during the course of the $\stackrel{\Phi}{\varnothing}$ disease. The absence of a disease specific chromosomal marker means that methods which $\vec{\circ}$ use PCR to detect clonal rearrangements at the immunoglobulin heavy chain $(\operatorname{IgH})$ locus are $\vec{\omega}$ the most applicable for the assessment of residual disease. A number of methods have? been described to date; the majority use a con- $\overrightarrow{0}$ sensus primer from the $\mathrm{JH}$ region along with another primer from a variety of locations along the $\mathrm{VH}$ region (fig 1). The mosto commonly used technique was described by Brisco et $a l^{1}$; it utilises another consensus $\gg$ primer from the third framework region. Alter-ce native techniques utilise either six $\mathrm{VH}$ family specific primers from the first framework $\vec{\varphi}$ region $^{2}$ or a single consensus primer from the second framework region. ${ }^{34}$ A Framework 1 . consensus ( $\mathrm{Fr} 1 \mathrm{con}$ ) technique has been described recently; it utilises a single consensus primer which binds to a highly conserved site located approximately 30 bases upstream from $\Phi$ the Fr1f binding site. ${ }^{5}$

The ability to demonstrate clonal IgH윽 rearrangements varies between lymphoprolif- $\supset$ erative disorders and is also dependent on the PCR strategy used. Yields of up to $90 \%$ have been reported in acute lymphoblastic leukaemia (ALL) and chronic lymphocytic leukaemia 3 $(\mathrm{CLL})^{4}{ }^{6}$ but significantly lower yields have been reported in multiple myeloma and follicular lymphoma. ${ }^{17}$ The detection rate in 0 multiple myeloma and follicular lymphoma? may be improved by utilising the Fr2 and Fr1음 con PCR techniques. ${ }^{3-5}$ It has also been suggested recently that the failure to demon-os strate clonal rearrangements may be due to the $N$ fact that some $\mathrm{JH}$ primers have insufficient homology at their $3^{\prime}$ ends with $\mathrm{JH} 3$ and $\mathrm{JH} 6{ }^{\circ}$ segments. ${ }^{5}$ It is clearly desirable to use theo techniques which maximise the yield of clonal rearrangements; this is largely unknown in multiple myeloma as the majority of studies to $\frac{0}{0}$ date have looked predominantly at ALL and $\overrightarrow{\mathbb{D}}$ CLL or have looked at insufficient numbers of $\frac{}{\mathbb{P}}$ patients with multiple myeloma. In this study 2 we have therefore assessed all four IgH PCR strategies in a large series of patients and haveô also assessed the effect of $\mathrm{JH}$ primer selection on the ability to demonstrate clonal rearrange-흘 ments.

\section{Methods}

All the patients included in this study were diagnosed as having multiple myeloma by standard criteria and monoclonality was confirmed by the presence of circulating parapro- 


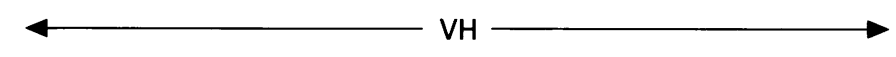

Fr1

Fr2

$\mathrm{Fr} 3$

Fr4

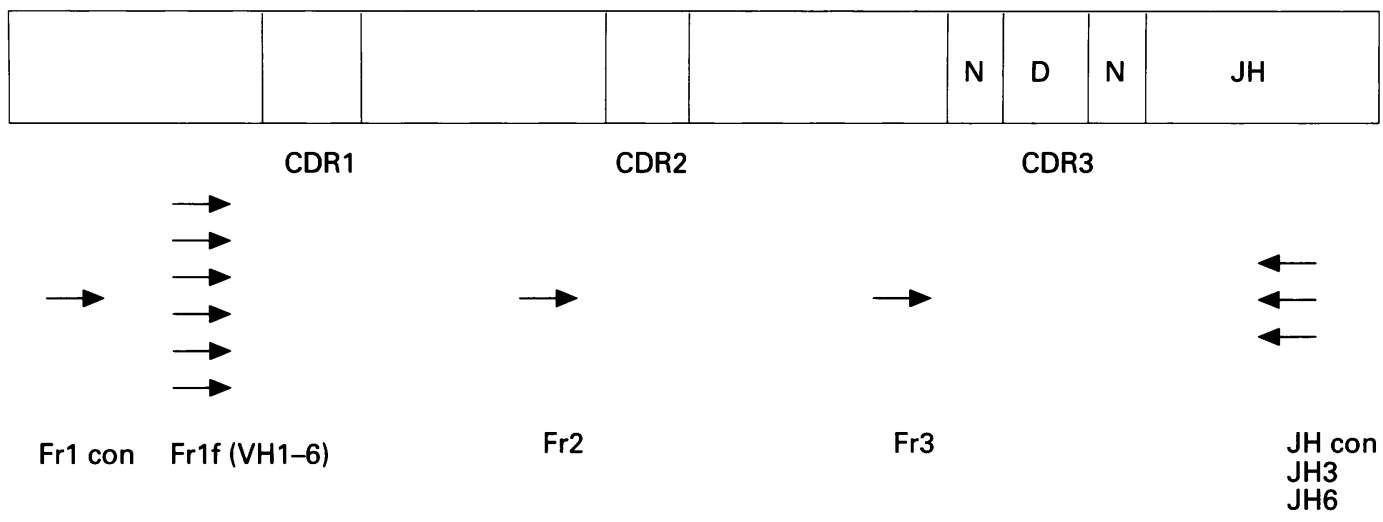

Figure 1 Schematic representation of a rearranged IgH gene. The primer binding sites for each of the four PCR techniques used in the present study are indicated.

tein or monoclonal cytoplasmic immunoglobulin, or both, within the bone marrow plasma cells. High molecular weight DNA was obtained from diagnostic bone marrow samples (containing at least $10 \%$ plasma cells) by proteinase $\mathrm{K}$ digestion, phenol/chloroform extraction and cold ethanol precipitation. The Fr3 PCR was assessed in 127 cases and the Fr1f PCR in 98. In addition, 44 cases were assessed by Fr3, Fr1f, Fr1 con and Fr2 PCR techniques. $\mathrm{JH}$ primer selection was also assessed such that each PCR technique was performed twice for each of the 44 cases using either the $\mathrm{JH}$ consensus primer alone or an equimolar mixture of $\mathrm{JH}$ consensus, $\mathrm{JH} 3$ and JH6 primers.

The primers used are outlined in table 1 ; the JH primers were $5^{\prime}$ end labelled via an aminohexyl link with a green fluorochrome. PCR reactions were performed in $25 \mu \mathrm{l}$ volumes with $1 \mu \mathrm{g}$ of DNA, 10 picomoles of sense and antisense primer in buffer containing $10 \mathrm{mM}$ Tris ( $\mathrm{pH}$ 9.1), $500 \mathrm{mM} \mathrm{KCl}, 0.25 \mathrm{mM}$ each $\mathrm{dNTP}$, and $1.5 \mathrm{mM} \mathrm{MgCl} \mathrm{m}_{2}$. When the mixture of $\mathrm{JH}$ primers was used, 5 picomoles of each was included per PCR reaction. The reaction mixtures were held at $95^{\circ} \mathrm{C}$ prior to the addition of 1 unit of Taq polymerase (SuperTaq, HT Biotechnology Ltd., Cambridge, UK). Conditions for Fr3 PCR amplification were: $95^{\circ} \mathrm{C}$ for one minute, $60^{\circ} \mathrm{C}$ for one minute and 30 seconds for a total of 35 cycles followed by 10 minutes at $72^{\circ} \mathrm{C}$. Conditions for Fr1f, Fr1 con and Fr2 PCR amplification

Table 1 Primer sequences used in each PCR strategy

\begin{tabular}{ll}
\hline Primer & Sequence \\
\hline Fr3 & 5' CCGAGGACACGGC(C/T)(C/G)TGTATTA CTG 3' \\
Fr1f: VH1 & 5' CCTCAGTGAAGGGTCTCCTGCAAGG 3' \\
VH2 & 5' GAGTCTGGTCCTGCGCTGGTGAAA 3' \\
VH3 & 5' GGTCCCTGAGACTCTCCTGTGCA 3' \\
VH4 & 5' TTCGGA(G/C)ACCCTGTCCCTCACCT 3' \\
VH5 & 5' GAGGTGAAAAAGCCCGGGAGTCT 3' \\
VH6 & 5' CCTGTGCCATCTCCGGGGACAGTG 3' \\
Fr1 con & 5' AGGTGCAGCTG(G/C)(A/T)G(G/C)AGTC(G/AT)GG 3' \\
Fr2 & 5' TGG(A/G)TCCG(A/C/G)CAG(G/C)C(T/C)CC(N)GG 3' \\
JH con & 5' ACCTGAGGAAGACGGTGACCAGGGT 3' \\
JH3 & 5' TACCTGAAGAGACGGTGACCATTGT 3' \\
JH6 & 5' ACCTGAGGAGACGGTGACCGTGGT 3' \\
\hline
\end{tabular}

were: $95^{\circ} \mathrm{C}$ for one minute followed by $68^{\circ} \mathrm{C}$ for one minute (reduced in consecutive cycles by $1^{\circ} \mathrm{C}$ to $62^{\circ} \mathrm{C}$ ) and $72^{\circ} \mathrm{C}$ for one minute for a total of 35 cycles, followed by $72^{\circ} \mathrm{C}$ for 10 minutes. The products of PCR amplification were then analysed as follows: $1 \mu \mathrm{l}$ of each PCR product was added to $4 \mu \mathrm{l}$ of loading mixture consisting of $3 \mu \mathrm{l}$ formamide, $0.5 \mu \mathrm{l}$ red fluorochrome labelled size standard (GS 2500 ROX, Applied Biosystems) and $0.5 \mu \mathrm{l}$ dextran blue. Each mixture was then denatured at $95^{\circ} \mathrm{C}$ for three minutes and held at $4^{\circ} \mathrm{C}$ prior to loading onto $6 \%$ polyacrylamide gels, which were then analysed using an Applied Biosystems automated DNA sequencer (model 373A). The associated collection and analysis software is able to convert the gel image for each sample into an "electrophoretogram" which consists of number of peaks whose height correspond to the intensity of fluorescence on the gel. A polyclonal pattern appears as a number of peaks separated by 3 base pairs (bp) and arranged in a normal distribution, whereas clonal rearrangements appear as distinct peaks. The software is able to size rearrangements consistently to within a single bp and is also able to size each peak within a fingerprint, which greatly facilitates the identification of clonal rearrangements of known size when they are present within a polyclonal background. Sensitivity was assessed to be at least $10^{-3}$ for each of the four techniques and control PCRs were not performed as polyclonal fingerprints were demonstrable in all "negative" samples.

\section{Results}

In total, 127 bone marrow samples (containing a minimum of $10 \%$ plasma cells) were analysed by using the Fr3 PCR. Clonal rearrangements were demonstrated in $71(56 \%)$. Single rearrangements predominated but biclonal rearrangements were seen in five cases. Rearrangements ranged from 87 to $173 \mathrm{bp}$ in size (normal range 85-140 bp) with the vast majority $(96 \%)$ lying within the normal fingerprint. This, in our experience, is in stark contrast to ALL were multiple rearrangements occur in 
Table 2 VH gene usage

\begin{tabular}{lcc}
\hline VH family & Multiple myeloma & Normal \\
\hline VH1 & $11 / 57(19 \%)$ & $29 \%$ \\
VH2 & $6 / 57(11 \%)$ & $5 \%$ \\
VH3 & $28 / 57(49 \%)$ & $49 \%$ \\
VH4 & $9 / 57(16 \%)$ & $14 \%$ \\
VH5 & $3 / 57(5 \%)$ & $2 \%$ \\
VH6 & $0 / 57(0 \%)$ & $1 \%$ \\
\hline
\end{tabular}

A total of 57 clonal rearrangements were demonstrated in $52 / 98$ cases analysed with the Fr1f PCR (biallelic

rearrangements were seen in five of 52 positive cases). There is no obvious bias in the $\mathrm{VH}$ families used as has been reported no obvious bias in the

Table 3 Summary of results obtained with the four IgH PCR strategies in 44 cases of multiple myeloma

\begin{tabular}{lll}
\hline PCR & fH con & fH mix \\
\hline Fr3 & $26 / 44(59 \%)$ & $27 / 44(61 \%)$ \\
Fr1f & $26 / 44(59 \%)$ & $28 / 44(63 \%)$ \\
Fr3 + Fr1f & $34 / 44(77 \%)$ & $36 / 44(82 \%)$ \\
Frl con & $21 / 44(48 \%)$ & $22 / 44(50 \%)$ \\
Fr2 & $21 / 44(48 \%)$ & $23 / 44(52 \%)$ \\
Fr1 con + Fr2 & $28 / 44(63 \%)$ & $30 / 44(68 \%)$ \\
Total & $35 / 44(80 \%)$ & $37 / 44(84 \%)$ \\
\hline
\end{tabular}

Each PCR technique was performed twice for each of the 44 cases using the $\mathrm{JH}$ consensus primer alone and an equimolar mixture of $\mathrm{JH}$ consensus, $\mathrm{JH} 3$ and $\mathrm{JH} 6$ primers $(\mathrm{JH}$ mix). Note mixture of $\mathrm{JH}$ consensus, $\mathrm{JH} 3$ and $\mathrm{JH} 6$ primers $(\mathrm{JH}$ mix
that the Fr1 con and $\mathrm{Fr} 2 \mathrm{PCR}$ techniques have inferior that the Fr1 con and Fr2 PCR techniques have inferio
detection rates compared with the Fr3 and Fr1f PCR techniques. The Fr2 PCR did, however, demonstrate a techniques. The Fr2 PCR did, however, demonstrate a
rearrangement in one case negative by both Fr3 and Frlf. Clonal rearrangements were detected in two additional cases by using the $\mathrm{JH}$ mix; one case became positive by $\mathrm{Fr} 3$, Frlf and Fr2 and the other by Frlf, Fr1 con and Fr2.

$40 \%$ with many lying outside the normal range (data not shown).

Diagnostic bone marrow specimens from 98 cases were also analysed with the Frlf PCR, clonal rearrangements being demonstrable in $52(53 \%)$ of 98 cases. Fr1f rearrangements ranged from 294 to $368 \mathrm{bp}$ in size with $96 \%$ lying within the normal fingerprint (the normal range varies with each $\mathrm{VH}$ family). Biallelic rearrangements were seen in five cases. There did not seem to be any bias in $\mathrm{VH}$ gene usage (table 2) such that VH3 rearrangements predominated (49\%) and $\mathrm{VH} 5$ and $\mathrm{VH} 6$ rearrangements were rare $(5 \%$ and $0 \%$, respectively).

A combination of both Fr3 and Fr1f PCR techniques demonstrated definitive clonal rearrangements in $92(75 \%)$ of 122 cases (five cases were negative by Fr3 PCR and had insufficient DNA for analysis by Fr1f PCR). Fr1 con and Fr2 PCR techniques were also assessed in 44 cases of confirmed multiple myeloma (table 3). In these cases the Fr3 and Frlf PCRs had demonstrated rearrangements in $26(59 \%)$ cases with a combined yield of 34 (77\%). Both the Fr1 con and the Fr2 techniques had inferior pick up rates, demonstrating clonal rearrangements in $21(48 \%)$ of 44 cases with a combined yield of $28(63 \%)$. The Fr2 PCR did, however, demonstrate a clonal rearrangement in one case which was negative by both Fr3 and Fr1f.

In six cases the clonal IgH rearrangement could only be demonstrated by Fr1f PCR. The Fr1 con PCR yielded rearrangements of 349-394 bp in size (normal range 350-405 bp) and the Fr2 rearrangements of 192-285 bp (normal range 240-285 bp). In both instances $98 \%$ of rearrangements lay within the normal fingerprint.
By utilising a mixture of $\mathrm{JH}$ primers $\mathrm{JH}$ con, JH3 and JH6) rearrangements were demonstrated in a further two cases: one became positive by Fr3, Fr1f and Fr2 and the other by Fr1f, Fr1 con and Fr2.

\section{Discussion}

The sequence of clonal IgH rearrangements in multiple myeloma (unlike ALL) remain unchanged throughout the course of the disease, ${ }^{10}$ making it an ideal marker for studies of residual disease. PCR techniques which demonstrate such rearrangements either on the basis of their size or specific sequence are being increasingly used in the study of multiple myeloma. It has been possible with such techniques to demonstrate clonal rearrangements (identical with those seen in diagnostic bone marrow samples) in the peripheral blood of the majority of patients at diagnosis. ${ }^{11}{ }^{12}$ Studies have also demonstrated clonal rearrangements $\vec{c}$ in peripheral blood progenitor cell collections ${ }^{13}{ }^{14}$; and also that these may be eliminated by purging strategies such as CD34 selection or cell sorting. ${ }^{14-16}$ The presence of residual disease has also been assessed in patients who have undergone allogeneic ${ }^{17}$ and autologous ${ }^{18}$ transplantation.

It is clearly advantageous to be able to demonstrate IgH rearrangements in as many patients as possible. In this study we have compared the ability of the four commonly used PCR techniques to demonstrate clonal IgH rearrangements in multiple myeloma. We were able to demonstrate clonal rearrangements in $56 \%$ of cases with the Fr3 PCR and 53\% with the Frlf PCR. Barker et $a l^{19}$ were able to demonstrate clonal Frlf rearrangements in 23/28 cases but this detection rate has not been achieved by others. ${ }^{5}$ However, by combining both techniques we were able to demonstrate definitive clonal rearrangements in $75 \%$ of cases. Recent reports have suggested that a greatly improved yield (up to $100 \%$ of cases) could be obtained with the Fr2 and Fr1 con PCR techniques. ${ }^{3-5}$ These studies involved small numbers of patients with multiple myeloma and we were unable to confirm their findings; both techniques detected clonal rear- 응 rangements in $21(48 \%)$ of 44 cases with a N combined yield of $28(63 \%)$. We therefore rec- N ommend the routine use of both the Fr3 and 0 Fr1f PCR techniques to demonstrate clonal 0 IgH rearrangements in multiple myeloma. The $\mathbb{D}$ Fr1 con and Fr2 PCR techniques have signifi- ? cantly inferior pick up rates and do not detect 0 many additional rearrangements; the Fr2 PCR $\stackrel{\vec{D}}{\mathrm{D}}$ demonstrated one additional rearrangement in our series of 44 cases. Although the Fr1f technique requires six PCR reactions we believe it is important to use it as a significant number of $\delta$ rearrangements were only demonstrable by this technique. In addition, it has the added

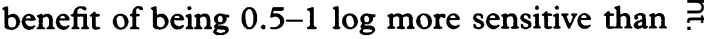
the Fr3 PCR in the majority of cases (data not shown). It has also been suggested recently that some $\mathrm{JH}$ consensus primers may lack homology at their $3^{\prime}$ ends with $\mathrm{JH} 3$ and $\mathrm{JH} 6$ segments. ${ }^{5}$ In this study we have assessed each PCR strategy with a JH consensus primer 
alone and combined with $\mathrm{JH} 3$ and $\mathrm{JH} 6$ specific primers. By utilising the mixture of $\mathrm{JH}$ primers it was possible to demonstrate rearrangements in two additional cases; one became positive by Fr3, Frlf and Fr2 and the other positive Fr1f, Fr1 con and Fr2.

It is clear therefore that it will not be possible to demonstrate clonal $\mathrm{IgH}$ rearrangements in approximately $20 \%$ of cases. This is likely to be due to the loss of $\mathrm{VH}$ primer binding sites secondary to somatic hypermutation which has been demonstrated by a number of investigators. ${ }^{102021}$ The extent of mutation seems to be greater in multiple myeloma with a median of $8 \%$ of nucleotides mutated compared with $2 \%$ in CLL and $4 \%$ in follicular lymphoma. ${ }^{22}$ Extensive mutation has also been demonstrated in $\mathrm{V}_{\text {kappa }}$ genes, again the extent of mutation seemed to be greater in multiple myeloma than in other lymphoproliferative disorders. $^{23}$

In this study we were able to demonstrate clonal Frlf rearrangements in $52(53 \%)$ of 98 cases. There did not seem to be any bias in the $\mathrm{VH}$ genes used such that $\mathrm{VH} 3$ rearrangements predominated, and VH5 and VH6 rearrangements were rare. This pattern, which has been reported previously in a smaller series of patients, ${ }^{19}$ is also seen in follicular lymphoma ${ }^{24}$ but not ALL and CLL where the over representation of $\mathrm{VH} 5$ and VH6 families is a well recognised phenomenon. ${ }^{689}$ In addition, certain specific $\mathrm{VH}$ genes-for example, $\mathrm{VH} 4-$ 21, VH4-18 and VH1 51P1, have been demonstrated to be used preferentially in ALL and CLL. ${ }^{25-27}$ Sequence data is more limited in multiple myeloma although it does seem that VH4-21 is not used preferentially. ${ }^{28}$ We conclude that clonal IgH rearrangements can be demonstrated in the majority of patients with multiple myeloma. It would seem that the Fr3 and Frlf PCR techniques provide the optimal combination demonstrating rearrangements in up to $80 \%$ of cases.

This work was supported by the Leukaemia Research Fund and the Yorkshire Cancer Research Campaign.

1 Brisco MJ, Tan LW, Orsborn AM, Morley AA. Development of a highly sensitive assay, based on the polymerase chain reaction, for rare B-lymphocyte clones in a polyclonal population. Br ₹ Haematol 1990;75:163-7.

2 Deanne M, Norton JD. Immunoglobulin gene "fingerprinting": an approach to analysis of B lymphoid clonality in ing": an approach to analysis of B lymphoid clonality in lymphoprol

3 Ramasamy I, Brisco M, Morley A. Improved PCR method for detecting monoclonal immunoglobulin heavy chain rearrangement in B cell neoplasms. $f$ Clin Patho 1992;45:770-5.

4 Diss TC, Peng H, Wotherspoon AC, Isaacson PG, Pan L. Detection of monoclonality in low grade B-cell lymphomas using the polymerase chain is dependent on primer selection and lymphoma type. $\mathcal{F}$ Pathol 1993;169:291-5.

5 Aubin J, Davi F, Nguyen-Salomon F, Leboeuf D, Debert C Taher M, et al. Description of a novel FR1 IgH PCR strategy and its comparison with three other strategies for the detection of clonality in B cell malignancies. Leukemia 1995;9:471-9.

6 Deanne MD, Norton JD. Immunoglobulin heavy chain variable region family usage is independent of tumour cell phenotype in human B lineage leukemias. Eur $\mathcal{F}$ Immunol 1990;20:2209-17.
7 Trainor KJ, Brisco MJ, Wan JH, Neoh S, Grist S, Morley AA. Gene rearrangements in B and T lymphoproliferative disease detected by the polymerase chain reaction. Blood 1991;78:192-8.

8 Mayer R, Logtenberg T, Strauchen J, Dimitriu-Bona A, Mayer L, Mechanic S, et al. CD5 and immunoglobulin V gene expression in B-cell lymphomas and chronic lymphocytic leukemia. Blood 1990;75:1518-24.

9 Kipps TJ, Carson DA. Autoantibodies in chronic lymphocytic leukemia and related systemic autoimmune disorders. Blood 1993;81:2475-87.

10 Ralph QM, Brisco MJ, Joshua DE, Brown R, Gibson J, Morley AA. Advancement of multiple myeloma from diagnosis through plateau phase to progression does not involve a new B-cell clone: evidence from the Ig heavy chain gene. Blood 1993;82:202-6.

11 Billadeau D, Quam L, Thomas W, Kay N, Greipp P, Kyle R, et al. Detection and quantitation of malignant cells in the peripheral blood of multiple myeloma patients. Blood 1992;80:1818-24

12 Corradini P, Voena C, Omede P, Astolfi M, Boccadoro M, Dalla-Favera $\mathrm{R}$, et al. Detection of circulating tumour cells in multiple myeloma by a PCR based method. Leukemia 1993;11:1879-82.

13 Bird JM, Bloxham D, Samson D, Marcus RE, Russell NH, Kelsey SM, et al. Molecular detection of clonally rearranged cells in peripheral blood progenitor cell harvests from multiple myeloma patients. $\mathrm{Br} 7 \mathrm{Haematol}$ 1994;88:110-16.

14 Owen RG, Haynes AP, Evans PA, Johnson RJ, Rawstron A, McQuaker $G$, et al. Detection of clonal immunoglobulin gene rearrangements in the peripheral blood progenitor cells of patients with multiple myeloma: the potential role of purging with CD34 positive selection. $\mathcal{F}$ Clin Pathol: $\mathrm{Mol}$ Pathol 1996;49:M112-17.

15 Schiller G, Vescio R, Freytes C, Spitzer G, Sahebi F, Lee M, et al Transplantation of CD34+ peripheral blood progenitor cells after high-dose chemotherapy for patients with tor cells after high-dose chemotherapy for patien.

16 Gazitt Y, Reading CC, Hoffman R, Wickrema A, Vesole $\mathrm{DH}$, Jaganath S, et al. Purified CD 34+Lin-Thy+stem cells do not contain clonal myeloma cells. Blood 1995;86:3819 .

17 Bird JM, Russell NH, Samson D. Minimal residual disease after bone marrow transplantation for multiple myeloma: evidence for cure in long term survivors. Bone Marrow Transplant 1993;12:651-4.

18 Corradini P, Voena C, Astolfi M, Ladetto M, Tarella C, Boccadoro $\mathrm{M}$, et al. High dose sequential chemoradiotherapy in multiple myeloma: residual tumour cells are detectable in bone marrow and peripheral blood cell harvests and after autografting. Blood 1995;85:1596-602.

19 Baker BW, Deanne M, Gilleece MH, Johnston D, Scarffe $\mathrm{JH}$, Norton JD. Distinctive features of immunoglobulin heavy chain variable region gene rearrangement in multiple myeloma. Leuk Lymphoma 1994;14:291-301.

20 Bakkus MHC, Heirman C, Van Riet I, Van Camp B, Thielemans K. Evidence that multiple myeloma Ig heavy chain VDJ genes contain somatic mutations but show no intraclonal variation. Blood 1992;80:2326-35.

21 Berenson JR, Vescio RA, Hong CH, Cao J, Kim A, Lee CC, et al. Multiple myeloma clones are derived from a cell in late B cell development. Curr Top Microbiol Immunol 1994; 194:25-33.

22 Vescio RA, Kieber-Emmons T, Lee JC, Wu CH, Cao J, Shin $\mathrm{J}$, et al. Immmunoglobulin heavy chain gene analysis proves that myeloma oncogenesis occurs late in B-cell proves that myeloma oncogenesis occurs late in B-cell
development. Proceedings of the Vth International Workdevelopment. Proceedings of the Vth International
shop on Multiple Myeloma. Section 3. 1995:19.

23 Wagner SD, Martinelli V, Luzzatto L. Similar patterns of Vk gene usage but different degrees of somatic mutation in hairy cell leukemia, prolymphocytic leukemia, Waldenstrom's macroglobulinemia and myeloma. Blood 1994; 83:3647-53.

24 Bahler DW, Campbell MJ, Hart S, Miller RA, Levy S, Levy R. Ig VH gene expression among human follicular lymphoma. Blood 1991;78:1561-8.

25 Deanne M, Norton JD. Preferential rearrangement of developmentally regulated immunoglobulin VH1 genes in human B-lineage leukaemias. Leukemia 1991;5:646-50.

26 Deanne M, Baker BW, Norton JD. Immunoglobulin VH4 gene usage in $\mathrm{B}$ lymphoid leukaemias. $\mathrm{Br} \mathcal{F} \mathrm{Haematol}$ 1993;84:242-9.

27 Maloum K, Magnac C, Pritsch O, Binet JL, Merle-Beral H, Dighiero G. Skewed rearrangement of the VH4-21 gene during pre-B acute lym

28 Rettig MB, Vescio RA, Cao J, Wu CH, Lee JC, Han E, et al. $\mathrm{VH}$ gene usage in multiple myeloma: complete absence of VH4-21 (VH4-34) gene [abstract]. Blood 1995;86(Suppl 1):60a. 